\title{
Performance Evaluation of Impulse Radio UWB Networks using Common or Private Acquisition Preambles
}

\author{
Ruben Merz, Jean-Yves Le Boudec \\ EPFL, School of Computer and Communication Sciences \\ \{ruben.merz, jean-yves.leboudec\}@epfl.ch
}

\begin{abstract}
-
For impulse-radio ultra-wide band (IR-UWB) networks without global synchronization, the first step for correct packet reception is packet detection and timing acquisition: Before recovering the payload of the packet, the destination must detect that the packet is on the medium and determine when exactly the payload begins. Packet detection and timing acquisition rely on the presence of an acquisition preamble at the beginning of each packet. How this preamble is chosen is a network design issue and it may have quite an impact on the network performance. A simple design choice of the network is to use a common acquisition preamble for the whole network. A second design choice is to use an acquisition preamble that is private to each destination. The throughput with the latter choice is likely to be much higher, albeit at the cost of learning the private acquisition preamble of a destination. In this paper, we evaluate how using a common or private acquisition preambles affects the network throughput. Our analysis is based on analytical modeling and simulations. Using our analytical model, we show that a private acquisition preamble yields a tremendous increase in throughput compared to a common acquisition preamble. The throughput difference grows with the number of concurrent transmitters and interferers. This result is confirmed by simulations. Furthermore, additional simulations on multi-hop topologies with TCP flows demonstrate that a network using private acquisition preambles has a stable throughput. On the contrary, using a common acquisition preamble exhibits the presence of a compounding effect similar to the exposed terminal issue in IEEE 802.11 networks: the throughput is severely degraded and complete flow starvation may occur.
\end{abstract}

\section{INTRODUCTION}

Emerging pervasive wireless networks with nodes embedded in everyday life objects have a size that ranges from a few dozen nodes to large-scale networks composed of hundreds of nodes. Their average data-rate is low, on the order of $1 \mathrm{Mbit} / \mathrm{s}$. They are packet based and have no global synchronization. Hence, the first step towards correct packet reception is packet detection and timing acquisition: Before recovering the payload of the packet, the destination of the packet must detect the packet on the medium and determine when exactly the payload begins. Notice that even if there exists a global synchronization

The work in this paper was partly presented at the IEEE International Conference on Ultra-wideband (ICUWB), Boston, September 2006 [1]. The work presented in this paper was supported (in part) by the National Competence Center in Research on Mobile Information and Communication Systems (NCCR-MICS), a center supported by the Swiss National Science Foundation under grant number 5005-67322, and by CTI contract No7109.2;1 ESPP-ES in the network, packet detection is still necessary. Packet detection and timing acquisition are performed on a per packet basis and both typically rely on the presence of an acquisition preamble at the beginning of each packet. How this preamble is chosen is a network design issue and, as we demonstrate in this article, it has an impact on the performance of the network.

In this paper, we evaluate how the choice of the acquisition preamble affects the throughput for networks using an impulse-radio ultra-wide band (IR-UWB) physical layer. This physical layer makes use of ultra-short duration (smaller than one nanosecond) pulses that yield ultra-wide bandwidth signals. They are characterized by a low duty-cycle (around $1 \%$ ) and extremely low power spectral densities [2]. The pulses are sent infrequently and, thanks to time-hopping (see Section II-A), the transmit time of each pulse is additionally randomized. As such, the IR-UWB physical layer is a multichannel physical layer where several users can share the medium concurrently. IR-UWB physical layers are especially attractive for low data-rate wireless communications, because they potentially combine low-power consumption, robustness to multipath fading and location/ranging capability. The IEEE 802.15.4a amendment [3] specifies an IR-UWB physical layer for the IEEE 802.15.4 standard [4], [5] that can operate over several bands of $500 \mathrm{MHz}$ (or $1.5 \mathrm{GHz}$ ) from approximately $3 \mathrm{GHz}$ to $10 \mathrm{GHz}$.

We compare two possible design choices. First, as in the IEEE 802.15.4a amendment, a simple design choice of the network is to have an identical and common acquisition preamble for the entire network. Second, as in [6], [7], another design choice is to have a private acquisition preamble per destination. For example, in [6], a source computes the acquisition preamble of its intended destination as a function of a unique identifier of the destination. Such an identifier can be, for instance, the MAC address.

The throughput of a network using private acquisition preamble is likely to be much higher than the throughput of a network using a common acquisition preamble. Indeed, in a network with a common acquisition preamble, a packet might contend during timing acquisition with packets sent by any node in the entire network. In contrast, with a private acquisition preamble, the contention is reduced to packets transmitted to the same destination. Of course, a private acquisition preamble comes with the cost of learning the 
acquisition preamble of the destination. Hence the throughput increase must be large (maybe larger than 100\%) in order to alleviate the associated costs ${ }^{1}$. And with digital hardware implementations, the use of private acquisition preambles is not more costly than a single common preamble [8]. In fact, a node does not need to listen to more than a few preambles [6]. Generally, a node needs to listen to its own preamble, the one from the destination and a preamble for the broadcast address.

For the performance evaluation of a network using either common or private acquisition preambles, we use two different approaches. Our first approach is to derive an analytical model to compute the saturation throughput (see Equations (15) and (16)), thus establishing an equivalent for an IR-UWB network of the celebrated Bianchis's formula [9]. In saturated conditions, a source has always a packet available to transmit and queueing at the source is ignored. Even though IRUWB networks are expected to be low-data rate networks, the performance in saturation conditions still matters. For instance, in case of sudden bursts of activity (detection of a fire in a building), it is important to ensure that the network is able to sustain the sudden load. Due to the inherent difficulty, the computation of the saturation throughput is solved for symmetric and homogeneous networks where all nodes are in range of each other. Our analytical model is built on a mean-field assumption and involves the resolution of a fixedpoint equation (see Section III). The mean-field assumption is equivalent to the decoupling approximation in [10].

Our second approach is to turn to ns-2 [11] simulations. First, they allow for the validation of the results obtained with our analytical model. Second, we can address more realistic scenarios with multi-hop topologies and TCP.

For both the analytical model and the ns-2 simulations, we assume that the underlying acquisition algorithm is the one in [12]. At the link layer, we assume that the DCC-MAC protocol [6] is used. The reason for choosing the acquisition algorithm in [12] is its robustness to multi-user interference (MUI). For DCC-MAC, the protocol is fully implemented in the ns-2 network simulator along with a model of an IR-UWB physical layer [13] (with the code fully available at [14]). More details on the physical layer and on the DCC-MAC protocol are given in Section II.

For both the analytical model and the ns-2 implementation, packet detection and timing acquisition is not modeled at the same level of details than the physical layer. Due to the time scale difference between events at the physical layer and events at the upper layers, the complexity is huge. Rather, we use the probability of missed detection and the probability of false alarm at the physical layer derived in [12] as inputs for a model of packet detection and timing acquisition at the link layer (see Section III-B). Furthermore, in the analytical model, we consider noise and MUI only during packet detection and timing acquisition. We expect that interference in the data transmission part will have little effect on our results, as we focus on timing acquisition. This is a reasonable assumption

\footnotetext{
${ }^{1}$ If TCP/IP is running, nodes have a unique identifier that must be known by neighboring nodes and the acquisition preamble can be easily generated from the unique identifier. Furthermore, IEEE 802.15.4 nodes have a unique EUI-64 identifier that could also be used to generate an acquisition preamble.
}

in low data-rate conditions with an optimal Rake receiver [15] at the physical layer where, with the addition of an error correcting code, the bit error rate can be negligible. In addition, in the case of unintentional packet acquisition (i.e. a packet not for the destination), we consider two options. With early discard, a destination drops the packet right after the header containing the hardware address. With late discard, the packet is fully received. This is often necessary, because a packet may have to be fully received in order to decode a possible checksum. Note that even in the case of private acquisition preambles, unintentional packet acquisition can occur due to noise and MUI [12].

Our contributions in this paper are the following. First, we developed an analytical model to compute the saturation throughput of an IR-UWB network. One novelty of our model, compared to previous work, is that it explicitly takes into account packet detection and timing acquisition. Our model can be used with different MAC layers or different acquisition algorithms. Then, using the model, we show that a private acquisition preamble offers a large throughput gain compared to a common acquisition preamble. Moreover, the throughput difference grows with the number of concurrent transmitters and interferers. Finally, with ns-2 simulations on multi-hop topologies with TCP flows, we demonstrate that a network using private acquisition preambles exhibits a stable throughput. On the contrary, using a common acquisition preamble exhibits the presence of a compounding effect similar to the exposed terminal issue in IEEE 802.11 networks: the throughput is severely degraded and complete flow starvation may occur.

The remainder of this paper is organized as follows. In Section II, we describe the system model and assumptions. We also give the necessary details on the underlying physical layer and on the DCC-MAC protocol. In Section III, we develop the analytical model to compute the saturation throughput of a symmetric and homogeneous UWB network. A summary of how to use our method to compute the saturation throughput can be found in Section III-C. The performance evaluation follows in Section IV. We discuss related work in Section V and conclude the paper in Section VI.

\section{SYSTEM MODEL: PHYSICAL LAYER AND MAC PROTOCOL}

In this section, we describe the underlying IR-UWB physical layer and give the necessary material for the DCC-MAC protocol in order to understand the modeling in Section III.

\section{A. Physical layer model: impulse-radio ultra-wide band with time-hopping}

IR-UWB physical layers make use of extremely short duration pulses ${ }^{2}$ that yield ultra-wide bandwidth signals (pulse duration $\leq 2 \mathrm{~ns}$ for a bandwidth $\geq 500 \mathrm{MHz}$ ). The pulses are sent infrequently, with a typical duty cycle around $1 \%$ for low data-rate systems. Due to stringent radio spectrum regulations because of their large bandwidth (e.g. overlap with

\footnotetext{
${ }^{2}$ Or short bursts of short pulses as in the IEEE 802.15.4a amendment.
} 
the frequencies of existing systems, for instance WiMAX frequencies), UWB systems are also characterized by extremely low power spectral densities.

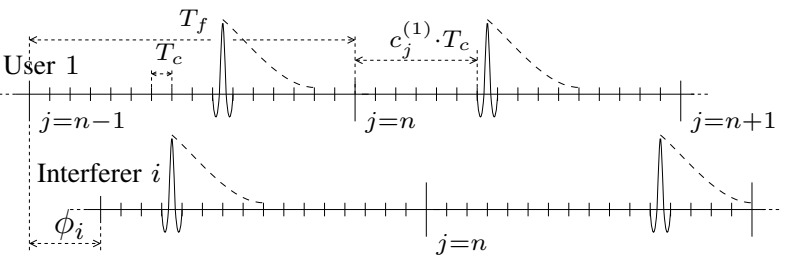

Fig. 1. Impulse-radio UWB physical layer with time-hopping: $c_{j}^{(i)}$ denotes the time-hopping sequence of user $i$ and $\phi_{i}$ is the delay between interferer $i$ and the user of interest (user 0 ). The dashed curve following each pulse represents the multipath propagation channel.

The IR-UWB physical layer model [2] is illustrated in Figure 1 and explained in the following. Time is divided into frames of duration $T_{f}$. There is only one pulse of duration $T_{p}$ transmitted per frame. The multi-channel capability of IRUWB physical layers stems from time-hopping: A frame is further subdivided into non-overlapping chips of duration $T_{c}$, where $T_{c} \geq T_{p}$. For each frame, these chips define the possible locations for the transmission of a pulse. A so-called (pseudorandom) time-hopping sequence (THS) indicates which chip to choose in each frame for the transmission of a pulse. Another distinctive characteristic of UWB systems is their multipath resolvability. In indoor environments, multipath occurs due to reflection, refraction and scattering of radio waves by surrounding structures: The transmitted signal reaches the receiver by more than one path [16]. But a direct consequence of the short duration of the pulses is that the multiple paths may be separately identified [17].

The acquisition preamble, appended at the beginning of each packet, is also generated using a THS. A common acquisition preamble implies the use of the same THS by all transmitters. The packet detection and timing acquisition algorithm uses the knowledge of the THS that generated the acquisition preamble to distinguish the acquisition preamble from noise and other concurrent transmissions that use different acquisition preambles. In the case of concurrent transmissions with a common acquisition preamble, the algorithm in [12] allows for a successful timing acquisition with one of the concurrent signals. The exact functioning of a packet detection and timing acquisition algorithm is out of the scope of this article. The interested reader can consult [15].

Unlike narrow-band systems, the collisions of packets from different transmitters do not fully destroy the underlying radio signals. The radio signals from the different transmitters can be recovered. In fact, if several users transmit concurrently with distinctive THS, occasional pulse collisions will occur between the concurrent signals that can be easily corrected by an error correcting code as demonstrated in [6]. Hence, the IR-UWB physical layer with time-hopping allows several users to share the medium concurrently.

\section{B. The DCC-MAC protocol}

DCC-MAC [6] is a MAC protocol for IR-UWB networks. It is designed to fully take advantage of the specifics of IR-
UWB physical layers and to operate efficiently on multi-hop networks. The goal of a MAC protocol is (1) to make sure that several senders do not attempt to communicate simultaneously with one destination and (2) to manage interference. This is traditionally solved in narrow-band networks by a carrier sensing scheme and the use of an RTS/CTS exchange. However, due to very low power emission, carrier sensing is hardly possible in IR-UWB networks. In addition, it is optimal for IR-UWB networks to allow concurrent transmissions to occur and to adapt the rate at the physical layer to the level of interference [18], [19]. Hence, DCC-MAC does not use RTS/CTS: It solves the issue of concurrent access at a destination with the combination of a careful selection of acquisition preambles to listen to, a careful selection of timer values, a binary exponential backoff algorithm as in 802.11 [20], and the use of a so-called SIGIDLE packet. The SIGIDLE packet is sent by a source upon the completion of a data transmission. It is used to tell neighboring sources that the node is now available for receiving data transmissions. For interference management, sources adapt to the level of interference at a destination with rate adaptation: sources dynamically change the rate of the channel code used at the physical layer on a per packet basis. A typical data exchange with DCC-MAC consists of a packet transmission from the source to the destination, an ACK from the destination and a SIGIDLE packet from the source. DCC-MAC can be used with both private and common acquisition preambles.

DCC-MAC is completely described in [6] where its performance in various scenarios has been thoroughly evaluated. It is fully implemented in the ns-2 network simulator along with a model of an IR-UWB physical layer [13]. The code is fully available at [14].

\section{A SATURATION THROUGHPUT ANALYSIS OF A UWB NETWORK WITH PACKET DETECTION AND TIMING ACQUISITION}

In this section, we compute the saturation throughput of a UWB network. In the following we give the problem description and important modeling assumptions.

1) Saturation throughput analysis and mean-field assumption: We begin by defining a few symbols:

- $\lambda_{0}^{(i)}$ is the saturation throughput of a source $i$ in packets per second.

- $\lambda^{(i)}$ is the rate of packet transmission attempts per second. Note that $\lambda^{(i)} \geq \lambda_{0}^{(i)}$, because $\lambda^{(i)}$ comprises successful packet transmissions and packet retransmissions.

- $p_{\text {acq }}^{(j)}$ is the average probability of correct packet detection and timing acquisition at a destination $j$.

- $S$ and $D$ are the total number of sources and destinations in the network, respectively.

Generally, finding the exact saturation throughput of every source is a highly difficult problem to solve [10]. In fact, we have to model the interactions of each node with every other node. Therefore, in order to keep the analysis tractable we make the following two assumptions:

1) The network is symmetric and homogeneous. Every destination has the same number of sources. 
2) We make a mean-field assumption [21] where we assume that all sources have an identical and independent behavior. Hence $\lambda_{0}^{(i)}=\lambda_{0}$ and $\lambda^{(i)}=\lambda$ for $i=$ $0, \ldots, S-1$, and $p_{\text {acq }}^{(j)}=p_{\text {acq }}$ for $j=0, \ldots, D-1$. The mean-field assumption is also known as the decoupling approximation in [10].

The first assumption also implies that all stations use the same physical layer and the same MAC layer (in our case the DCCMAC protocol). Second, we assume that in a saturated regime, the network model is ergodic. Indeed, there is no queueing and every source waits until a packet is successfully transmitted before attempting the transmission of a new packet. Finally, we break our general problem, of finding the saturation throughput, into two subproblems.

1) Given a source and its intended destination, the saturation throughput $\lambda_{0}$ of the source depends on the probability of successful packet acquisition $p_{\text {acq }}$ at the destination. Hence, our first subproblem is to compute $\lambda_{0}\left(\right.$ and $\lambda$ ) given $p_{\text {acq }}$ i.e. $\left[\lambda_{0}, \lambda\right]=f\left(p_{\text {acq }}\right)$. We solve this problem in Section III-A.

2) In the second subproblem, we have a receiver with several sources with saturation throughput $\lambda_{0}$ and attempt rate $\lambda$. We want to compute $p_{\text {acq }}$ i.e. $p_{\text {acq }}=g\left(\lambda_{0}, \lambda\right)$. We solve this problem in Section III-B.

Hence, the saturation throughput is given by $f(x)$ where $x$ is the solution of the fixed point equation

$$
g(f(x))-x=0, \quad x \in[0,1] .
$$

We solve the fixed point equation numerically. Section III-A explains how to solve the first subproblem in order to compute the values of $\lambda_{0}$ and $\lambda$ given $p_{\text {acq. }}$.

A. Computing $\lambda_{0}$ and $\lambda$ as a function of $p_{\text {acq }}$ : modeling the DCC-MAC protocol

1) Modeling the DCC-MAC protocol: The DCC-MAC protocol uses both a rate adaptation algorithm and a binary exponential backoff (Section II-B). In the modeling, we assume that DCC-MAC uses a fixed rate. Hence, the behavior of DCCMAC depends solely on the binary exponential backoff and can be modeled by a discrete-time, homogeneous, Markov chain [10]. We will use this Markov chain to compute both $\lambda_{0}$ and $\lambda$ given $p_{\text {acq }}$.

2) The retransmission Markov chain $X_{n}$ : Let $X_{n}$ be the retransmission state of a station (see Figure 2(a)) and $R$ the maximum number of retransmissions before a packet is dropped. The Markov chain $X_{n}$ has $R+2$ states (numbered from 0 to $R+1$ ): a packet transmission attempt always initiates and finishes in state 0 . A packet retransmission corresponds to a transition from state $i$ to $i+1$. A successful packet transmission corresponds to a transition from any state $i=0, \ldots, R$ to the state 0 . Finally, the packet is dropped if state $R+1$ is reached. The transition probabilities are the following:

$$
\begin{array}{ll}
p_{X}(i, i+1)=1-p_{\text {acq }}=p_{\text {fail }}, & i=0, \ldots, R \\
p_{X}(i, 0)=p_{\text {acq }}, & i=0, \ldots, R \\
p_{X}(R+1,0)=1 &
\end{array}
$$

where $p_{X}(i, j)=\mathbb{P}\left(X_{n+1}=j \mid X_{n}=i\right)$. The stationary distribution of $X_{n}$ is

$$
\pi_{X}(i)=\frac{p_{\text {acq }}\left(1-p_{\text {acq }}\right)^{i}}{1-\left(1-p_{\text {acq }}\right)^{R+1}}, \quad i=0,1, \ldots, R+1
$$

where we used $\sum_{k=0}^{n} x^{k}=\frac{1-x^{n+1}}{1-x}$.

3) Using the Markov chain $X_{n}$ to compute $\lambda_{0}$ and $\lambda$ : Each packet transmission attempt corresponds to a trip on the chain $X_{n}$ starting in state 0 and returning back to the state 0 . The saturation throughput $\lambda_{0}$ can be computed by dividing the average number of successful packet transmissions per trip by the average duration of a trip. Similarly, the attempt rate $\lambda$ can be computed by dividing the average number of packet transmission attempts per trip by the average duration of a trip.

For a trip from state 0 back to state 0 , we define three random variables:

- $N_{\mathrm{s}}$ is the number of successful packet transmissions per trip. Observe that $N_{\mathrm{s}}$ is equal to 0 or 1 .

- $N_{\mathrm{a}}$ is the number of packet transmission attempts per trip. $N_{\mathrm{a}}$ can take values in the range $[1,2, \ldots, R]$.

- $T$ is the duration of a trip.

To figure out the average of $N_{\mathrm{s}}$ per trip, the key observation is that a successful packet transmission corresponds to a transition from any state $i=0,1, \ldots, R$ to state 0 . Hence, in order to compute the average of $N_{\mathrm{s}}$ per trip, we need to compute the average number of transitions per trip from any state $0,1, \ldots, R$ to the state 0 . Likewise, a packet transmission attempt corresponds to a transition from state $i$ to $i+1$. Consequently, to compute the average of $N_{\mathrm{a}}$ per trip, we need to compute the average length of a trip on the chain $X_{n}$ before a transition back to state 0 (excluding the transition to state 0 ). Finally, to compute the average of $T$ per trip, notice that each state transition on the chain $X_{n}$ corresponds to a succession of events in the underlying MAC protocol with a given duration. For instance, a transition from state 0 to state 1 corresponds to the duration of a failed packet transmission. This duration comprises the duration of a packet transmission, the expiration of one or more timers, and the average backoff time in stage 1 .

More formally, in addition to the transition probabilities, we define $m(i, j)$ to be the cost of a transition from state $i$ to state $j$. Depending on whether we compute the average of $N_{\mathrm{s}}, N_{\mathrm{a}}$, or $T$ per trip, we assign different values to $m(i, j)$. For instance, for $N_{\mathrm{s}}$, we set $m(i, 0)=1$ for $i=0, \ldots, R$ and 0 otherwise. Then, we compute the average cost of a trip from the state 0 back to the state 0 with the proper values for $m(i, j)$. The cost of a particular trip is simply the sum of the cost of each transition in this trip.

The following two definitions formalize the content of the previous paragraphs.

Definition 1 (Time of first return to state 0): Let's assume that $X_{0}=0$ (i.e. the state of the Markov chain $X_{n}$ is 0 at time 0 ), then

$$
\tau_{1}=\inf \left\{n \geq 1 \mid X_{n}=0\right\}
$$

is the time of first return to state 0 . 
Definition 2 (Expected cost of a trip from state 0 to 0 ): Using the previous definition, the expected cost of a trip from state 0 back to state 0 is

$$
\mathbb{E}\left(\sum_{n=1}^{\tau_{1}} m\left(X_{n-1}, X_{n}\right) \mid X_{0}=0\right) .
$$

Therefore, the key to compute $\lambda_{0}$ and $\lambda$ is

1) To properly assign costs $m(i, j)$ to the transitions (see Equations (6), (7), and (8) for $N_{\mathrm{s}}, N_{\mathrm{a}}$, and $T$ respectively).

2) To compute (5) with the proper costs depending on whether we want to find the average of $N_{\mathrm{s}}, N_{\mathrm{a}}$, or $T$.

In the following subsections, we first define the costs for $N_{\mathrm{s}}$, $N_{\mathrm{a}}$, and $T$. Then, we explain how to compute (5) using Palm calculus [22], [23]. And we finally apply (5) to compute $\lambda_{0}$ and $\lambda$.

4) Definition of the costs to compute $\lambda_{0}$ and $\lambda$ : For $N_{\mathrm{s}}$, we must compute (5) with the the costs

$$
m_{N_{\mathrm{s}}}(i, 0)=1, \quad i=0, \ldots, R
$$

and 0 otherwise. For $N_{\mathrm{a}}$, we use instead

$$
m_{N_{\mathrm{a}}}(i, i+1)=1, \quad i=0, \ldots, R
$$

and 0 otherwise. Finally, for $T$, the costs are

$$
\begin{array}{ll}
m_{T}(i, 0)=t_{\mathrm{acq}}+t_{\mathrm{tx}}, & i=0, \ldots, R \\
m_{T}(i, i+1)=t_{\mathrm{acq}}+t_{\text {fail }}(i), & i=0, \ldots, R \\
m_{T}(R+1,0)=t_{\text {drop }} &
\end{array}
$$

and 0 otherwise. The quantity $t_{\mathrm{acq}}+t_{\mathrm{tx}}$ corresponds to the duration of a packet acquisition and timing synchronization followed by a successful DATA exchange, $t_{\text {acq }}+t_{\text {fail }}(i)$ is the duration incurred by a failed packet acquisition, and $t_{\text {drop }}$ is the time taken when the backoff algorithm reaches the $(R+1)$-th state where the packet to be transmitted is dropped. As they are protocol specific, the details of $t_{\mathrm{acq}}, t_{\mathrm{tx}}, t_{\text {fail }}(i)$, and $t_{\mathrm{drop}}$ are given in Section IV. Still, note that $t_{\text {fail }}(i)$ depends on $i$, i.e. it depends on the particular retransmission state; typically, as the number of retransmissions increases, the size of the contention window for the backoff timer increases.

5) Computing the expected cost of a trip using Palm calculus: We begin by recalling what a Palm probability and a Palm expectation are. This allows us to reformulate (5). Then, we recall one of the central results of Palm calculus, which is the Palm inversion formula, and we use it to compute (5).

Definition 3 (Palm probability and Palm expectation):

Given an integer valued point process $T_{n}$ of rate $\Lambda$, the Palm probability $\mathbb{P}^{0}$ is the conditional probability given that $T_{0}=0$. Similarly, the Palm expectation $\mathbb{E}^{0}$ is the conditional expectation given that $T_{0}=0$.

We can take advantage of Palm calculus to rewrite (5) as follows,

$\mathbb{E}\left(\sum_{n=1}^{\tau_{1}} m\left(X_{n-1}, X_{n}\right) \mid X_{0}=0\right)=\mathbb{E}^{0}\left(\sum_{n=1}^{\tau_{1}} m\left(X_{n-1}, X_{n}\right)\right)$

Using the previous formula, we have

$$
\lambda_{0}=\frac{\mathbb{E}^{0}\left(N_{\mathrm{s}}\right)}{\mathbb{E}^{0}(T)}, \quad \lambda=\frac{\mathbb{E}^{0}\left(N_{\mathrm{a}}\right)}{\mathbb{E}^{0}(T)}
$$

where $\mathbb{E}^{0}(\mathcal{X})=\mathbb{E}^{0}\left(\sum_{n=1}^{\tau_{1}} m_{\mathcal{X}}\left(X_{n-1}, X_{n}\right)\right)$ for $\mathcal{X}=N_{\mathrm{s}}, N_{\mathrm{a}}$ or $T$.

Now, to compute (9), we use the following result, available in [22] or [23] (see also in these references for a precise definition of joint stationarity):

Lemma 1 (Palm inversion formula): Let $Y_{n}$ be a discretetime random process and $T_{n}$ an integer valued point process of rate $\Lambda$. If $T_{n}, Y_{n}$ is jointly stationary, then

$$
\Lambda \mathbb{E}^{0}\left(\sum_{s=1}^{T_{1}} Y_{s}\right)=\mathbb{E}\left(Y_{0}\right)
$$

Hence, in order to compute (9), we use the Palm inversion formula with $Y_{n}=m_{\mathcal{X}}\left(X_{n-1}, X_{n}\right)$ and $T_{n}=\tau_{n}$ (the times of visit to state 0 ). Consequently, equation (9) becomes

$$
\begin{array}{r}
\mathbb{E}^{0}\left(\sum_{n=1}^{\tau_{1}} m_{\mathcal{X}}\left(X_{n-1}, X_{n}\right)\right)=\frac{\mathbb{E}\left(m_{\mathcal{X}}\left(X_{n-1}, X_{n}\right)\right)}{\Lambda} \\
=\frac{\sum_{i} \pi_{X}(i) \sum_{j} p_{X}(i, j) m_{\mathcal{X}}(i, j)}{\pi_{X}(0)}
\end{array}
$$

for $i, j=0, \ldots, R+2$.

6) Using the expected cost to compute $\lambda_{0}$ and $\lambda$ : Now, using (11) and the appropriate values of the costs (6), (7), and (8), we can compute $\mathbb{E}^{0}\left(N_{\mathrm{s}}\right), \mathbb{E}^{0}\left(N_{\mathrm{a}}\right)$, and $\mathbb{E}^{0}(T)$. We have

$$
\begin{gathered}
\mathbb{E}^{0}\left(N_{\mathrm{s}}\right)=\frac{1}{\pi_{X}(0)} \sum_{i=0}^{R} \pi_{X}(i) p_{X}(i, 0)=p_{\mathrm{acq}} \frac{\sum_{i=0}^{R} \pi_{X}(i)}{\pi_{X}(0)}, \\
\mathbb{E}^{0}\left(N_{\mathrm{a}}\right)=\frac{1}{\pi_{X}(0)} \sum_{i=0}^{R} \pi_{X}(i) p_{X}(i, i+1)=p_{\text {fail }} \frac{\sum_{i=0}^{R} \pi_{X}(i)}{\pi_{X}(0)},
\end{gathered}
$$

and

$$
\begin{aligned}
\mathbb{E}^{0}(T) & =\frac{1}{\pi_{X}(0)}\left[p_{\text {acq }}\left(t_{\text {acq }}+t_{\mathrm{tx}}\right) \sum_{i=0}^{R} \pi_{X}(i)\right. \\
+ & \left.p_{\text {fail }} \sum_{i=0}^{R}\left(t_{\text {acq }}+t_{\text {fail }}(i)\right) \pi_{X}(i)+t_{\text {drop }} \pi_{X}(R+1)\right]
\end{aligned}
$$

Finally, we can use equations (12) to (14) to replace $\mathbb{E}^{0}\left(N_{\mathrm{s}}\right)$, $\mathbb{E}^{0}\left(N_{\mathrm{a}}\right), \mathbb{E}^{0}(T)$ in $(10)$, and $\sum_{i=o}^{R} \pi_{X}(i)=1-\pi_{X}(R+1)$ with $\pi_{X}$ from (3) to obtain equations (15) and (16) on the top of next page.

Notice that (15) and (16) are general results that allow us to calculate the saturation throughput of a station given $p_{\text {acq }}$. The details of the MAC protocol and of the backoff algorithm are abstracted in the values of $t_{\mathrm{acq}}, t_{\mathrm{tx}}, t_{\text {fail }}(i), t_{\mathrm{drop}}$, and in the stationary distribution of the chain $X_{n}$. Now that we have (15) and (16) for calculating $\lambda_{0}$ and $\lambda$, we can turn to the resolution of the second subproblem.

\section{B. Computing $p_{a c q}$ as a function of $\lambda_{0}$ and $\lambda$ : modeling packet detection and timing acquisition}

Remember that $p_{\text {acq }}$ is the average probability of proper packet detection and timing acquisition at any destination. We compute $p_{\text {acq }}$ as a function of $\lambda_{0}$ and $\lambda$. We begin by defining a few symbols: 


$$
\begin{aligned}
\lambda_{0} & =\frac{p_{\text {acq }}\left(1-\pi_{X}(R+1)\right)}{p_{\text {acq }}\left(t_{\mathrm{acq}}+t_{\mathrm{tx}}\right)\left(1-\pi_{X}(R+1)\right)+p_{\text {fail }} \sum_{i=0}^{R}\left(t_{\mathrm{acq}}+t_{\mathrm{fail}}(i)\right) \pi_{X}(i)+t_{\mathrm{drop}} \pi_{X}(R+1)} \\
\lambda & =\frac{p_{\text {fail }}\left(1-\pi_{X}(R+1)\right)}{p_{\text {acq }}\left(t_{\mathrm{acq}}+t_{\mathrm{tx}}\right)\left(1-\pi_{X}(R+1)\right)+p_{\text {fail }} \sum_{i=0}^{R}\left(t_{\mathrm{acq}}+t_{\mathrm{fail}}(i)\right) \pi_{X}(i)+t_{\mathrm{drop}} \pi_{X}(R+1)}
\end{aligned}
$$

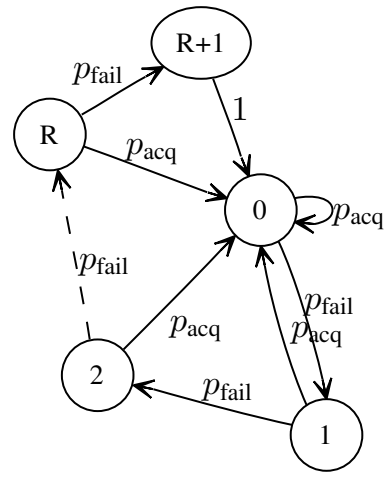

(a) $X_{n}$

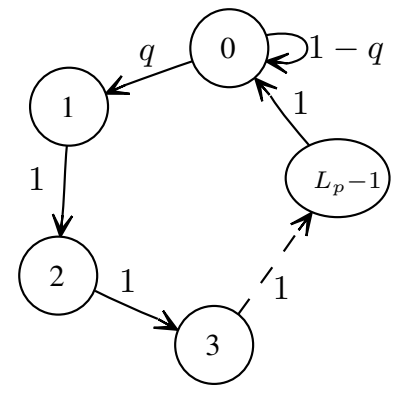

(b) $Z_{n}$
Fig. 2. Retransmission Markov chain $X_{n}$ (left) and transmission Markov chains $Z_{n}$ (right) with their transition probabilities. For $X_{n}$, note that $p_{\text {fail }}$ is simply $1-p_{\text {acq. }}$.

- $S_{\mathrm{D}}$ is the number of stations transmitting to the destination of interest. They use the same acquisition preamble than the destination of interest.

- $S_{\mathrm{I}}$ is the number of stations using the same acquisition preamble than the $S_{\mathrm{D}}$ ones but transmitting to another destination.

- $I$ is the number of stations using a different acquisition preamble.

1) Modeling packet detection and timing acquisition at a destination: A necessary condition for packet detection and timing acquisition to be achieved is that the destination not be busy. Following [13], the behavior of the physical layer of a station is modeled with four states:

- IDLE: The physical layer listens to the medium. We assume that a station never sleeps and is always available.

- SYNC: The physical layer believes it has detected a packet on the wireless medium and attempts to synchronize with the beginning of this packet.

- RECV: The physical layer receives the packet. It assumes that the physical layer has correctly detected that there is a packet and is synchronized with its beginning.

- SEND: The physical layer transmits a packet.

A station is considered busy when it is in the RECV or SEND state. A transition from the IDLE state to the SYNC state occurs whenever a packet from any of the $S_{D}$ or $S_{I}$ sources reaches the destination. Then, for a period of time equal to the length of the acquisition preamble of the packet that caused the transition, other packets from any of the $S_{D}+S_{I}-1$ sources may reach the destination and compete for acquisition. This period of time is called the "vulnerable period". At the end of the vulnerable period there are two possibilities: (1) one of the competing packets is acquired by the destination or (2) due to missed detection, the whole procedure fails. In the first case, a transition to the RECV state occurs. In the second case, the destination returns to the IDLE state. The probability that a missed detection occurs depends on the level of interference and on how many concurrent transmissions start from any of the $S_{D}+S_{I}+I-1$ other stations. In addition to increasing the level of interference, the packets from the $I$ stations with another acquisition preamble may spur occasional false acquisitions. Such false acquisitions trigger transitions to the RECV state and keep the destination busy as if it were receiving a packet from a valid station. Remember that we consider multi-user interference only during packet detection and timing acquisition.

Accordingly, the probability of packet acquisition at the destination of interest can be modeled as

$$
p_{\text {acq }}=\left(1-P_{\text {busy }}\right) \gamma
$$

where $P_{\text {busy }}$ is the probability that the destination is busy and $\gamma$ is the conditional probability that a packet from one of the $S_{D}+S_{I}$ sources is properly acquired without any missed detection given that the destination is not busy. False acquisitions are taken into account in the probability $P_{\text {busy }}$. Although it is not explicitly written in (17), both $P_{\text {busy }}$ and $\gamma$ depend on $\lambda_{0}$ and $\lambda$.

We first proceed with $\gamma$, the probability $P_{\text {busy }}$ is computed in the next Section. We model $\gamma$ as

$$
\gamma=\sum_{k=0}^{S_{\mathrm{D}}+S_{\mathrm{I}}-1} P_{k} \sum_{i=0}^{I} P_{i}\left[\frac{1}{k+1}\left(1-P_{\mathrm{MD}}^{(k, i)}\right)\right]
$$

where $P_{k}$ is the probability that there are $k$ concurrent transmissions from any of the $S_{D}+S_{I}-1$ other stations (with one transmission per source and the same acquisition preamble), and $P_{i}$ is the probability that there are $i$ concurrent transmissions from any of the $I$ stations (with a different acquisition preamble). The probability of missed detection $P_{\mathrm{MD}}^{(k, i)}$ depends on $k$ and $i$. The probability $1-P_{\mathrm{MD}}^{(k, i)}$ that there is no missed detection is uniformly divided by $k+1$ as there is the packet to be acquired and the $k$ competing packets from the $S_{D}+S_{I}-1$ other stations. Remember that we do not have an explicit model for $P_{\mathrm{MD}}^{(k, i)}$. Rather, we use numerical values from extensive simulations obtained with the packet detection and timing acquisition algorithm in [12]. The underlying assumption of (18) is that, thanks to the IRUWB physical layer, several packets can concurrently compete for packet detection and timing acquisition at a receiver. The probabilities $P_{k}$ and $P_{i}$ are modeled as

$$
P_{k}=\left(\begin{array}{c}
S_{\mathrm{D}}+S_{\mathrm{I}}-1 \\
k
\end{array}\right)\left(1-Q\left(L_{a c q}\right)\right)^{k} Q\left(L_{a c q}\right)^{S_{\mathrm{D}}+S_{\mathrm{I}}-1-k}
$$


and

$$
P_{i}=\left(\begin{array}{c}
I \\
i
\end{array}\right)\left(1-Q\left(L_{a c q}\right)\right)^{i} Q\left(L_{a c q}\right)^{I-i}
$$

where $Q\left(L_{a c q}\right)$ is the probability that a station does not start a packet transmission during the "vulnerable period" of length $L_{a c q}$ chips ( $L_{a c q}$ is equivalent to the length of the acquisition preamble in chips).

The set of equations (17) and (18) along with (21) to compute $P_{\text {busy }}$ and (33) to compute $Q\left(L_{a c q}\right)$ is the main result of this part. It allows for the calculation of $p_{\text {acq }}$ given $\lambda_{0}$ and $\lambda$. This set of equations is general: The equations model a physical layer with multi-channel capability. Note that the multiple channels need not be orthogonal. Actually, in the case of IR-UWB physical layers, the channels are created by the different THS (Section II-A) and are not orthogonal because of multipath propagation and asynchronous transmissions (Section II-A).

In the following, we describe first how to compute $P_{\text {busy }}$ and second how to compute $Q\left(L_{a c q}\right)$.

2) A model for $P_{\text {busy }}$ : There are three cases for which a destination can be busy:

- The destination is properly receiving a packet from any of the $S_{D}-1$ other competing sources.

- The destination is kept busy by a packet from any of the $S_{I}$ other sources with the same acquisition preamble but different destination.

- The destination is kept busy by the false acquisition of a packet from the $I$ sources with another acquisition preamble.

Remember that the packets from the $S_{I}$ stations are acquired with probability $p_{\text {acq }}$ (which takes into account the fact that the receiver could be busy). However, for the $I$ stations with a different THS, only a fraction $P_{\mathrm{FACQ}}$ of their packets is falsely acquired (assuming the destination is not busy). Hence, we model $P_{\text {busy }}$ as

$$
P_{\text {busy }}=\lambda_{0}\left(S_{\mathrm{D}}-1\right) t_{\mathrm{D}}+\lambda\left(p_{\text {acq }} S_{\mathrm{I}}+P_{\mathrm{FACQ}} I\right) t_{\mathrm{I}}
$$

where $t_{\mathrm{D}}$ is the time that a packet acquired from any of the $S_{\mathrm{D}}-1$ sources keeps the destination busy and $t_{\mathrm{I}}$ is the equivalent of $t_{\mathrm{D}}$ for the packets from the $S_{\mathrm{I}}$ and $I$ stations. Note that $t_{\mathrm{I}}<t_{\mathrm{D}}$ (see Section IV for their numerical values).

The fraction $P_{\mathrm{FACQ}}$ is expressed as

$$
P_{\text {FACQ }}=\frac{\lambda\left(1-P_{\text {busy }}\right) \Theta}{\lambda_{0}\left(S_{\mathrm{D}}-1\right)+\lambda p_{\text {acq }} S_{\mathrm{I}}+\lambda\left(1-P_{\text {busy }}\right) \Theta I}
$$

where $\Theta$ is a parameter that depends on the underlying physical layer and on the particular packet detection and timing acquisition method used.

3) Computing $Q\left(L_{a c q}\right)$ with the transmission Markov chain $Z_{n}$ : In this section, we explain how we obtain equation (33) to compute $Q\left(L_{a c q}\right)$, the probability that a station does not begin a packet transmission during the "vulnerable period" of length $L_{a c q}$ chips. This is the last quantity required to be able to calculate $p_{\text {acq }}$ given $\lambda_{0}$ and $\lambda$.

In order to determine $Q\left(L_{a c q}\right)$, we model the behavior of a station transmitting a packet at the physical layer with a discrete-time, homogeneous Markov chain $Z_{n}$. We begin by defining the transition probabilities of $Z_{n}$, then we relate $Z_{n}$ with $X_{n}$ (see Section III-A2), and finally we use $Z_{n}$ to obtain $Q\left(L_{a c q}\right)$.

Let $L_{p}$ be the number of chips per packet. Because our model must take into account the fact that a source can only transmit one packet at a time, $Z_{n}$ has $L_{p}$ states; state 0 is the state where the source waits for a new transmission to occur, the states 1 to $L_{p}-1$ are the states where a packet transmission is happening (see Figure 2(b)).

Let $q$ be the probability that a packet transmission starts. The transition probabilities of $Z_{n}$ are

$$
\begin{aligned}
& p_{Z}(0,0)=1-q \\
& p_{Z}(0,1)=q \\
& p_{Z}(i, i+1)=1, \quad i=1, \ldots, L_{p}-1 \\
& p_{Z}\left(L_{p}-1,0\right)=1 .
\end{aligned}
$$

The stationary distribution of $Z_{n}$ is

$$
\begin{aligned}
\pi_{Z}(0) & =\frac{1}{1+q\left(L_{p}-1\right)} \\
\pi_{Z}(i) & =\frac{q}{1+q\left(L_{p}-1\right)}, \quad i=1, \ldots, L_{p}-1 .
\end{aligned}
$$

In order to properly relate $X_{n}$ with $Z_{n}$, we need to relate $\lambda$ with $q$. Let $N_{p}$ be the number of packets transmitted during a time interval $t$. As $N_{p}=\lambda t$, we have $\pi_{Z}(1)=\frac{N_{p}}{t}=\lambda$. Therefore, using (25) for $i=1$ we obtain

$$
q=\frac{\lambda}{1-\lambda\left(L_{p}-1\right)} .
$$

Now that we have defined the transmission Markov chain $Z_{n}$, we can use $Z_{n}$ to compute $Q\left(L_{a c q}\right)$. Formally, we have

$Q\left(L_{a c q}\right)=\mathbb{P}\left(\right.$ A source does not visit state 1 in $\left.\left[0, L_{a c q}-1\right]\right)$

$$
=\mathbb{P}\left(Z_{0} \neq 1, Z_{1} \neq 1, \ldots, Z_{L_{a c q}-1} \neq 1\right) .
$$

In addition, we define

$$
Q\left(L_{a c q} \mid i\right)=\mathbb{P}\left(Z_{0} \neq 1, Z_{1} \neq 1, \ldots, Z_{L_{a c q}-1} \neq 1 \mid X_{0}=i\right) .
$$

Hence

$$
Q\left(L_{a c q}\right)=\sum_{i=0}^{L_{p}-1} Q\left(L_{a c q} \mid i\right) \pi_{Z}(i) .
$$

We already know how to obtain $\pi_{Z}(i)$ thanks to (25), but $Q\left(L_{a c q} \mid i\right)$ for $i=0,1, \ldots, L_{p}-1$ are still remaining. We compute them in an iterative fashion. By definition

$$
Q(0 \mid i)= \begin{cases}0 & \text { if } i=1 \\ 1 & \text { otherwise }\end{cases}
$$

and by construction

$$
Q\left(L_{a c q} \mid i\right)=\left\{\begin{array}{ll}
0 & \text { if } i=1 \\
\sum_{j \neq 1} p_{Z}(i, j) Q\left(L_{a c q}-1 \mid j\right) & \text { otherwise }
\end{array} .\right.
$$

Now, let's define the vector

$$
\vec{y}_{L_{a c q}}=\left[\begin{array}{lllll}
Q\left(L_{a c q} \mid 0\right) & 0 & Q\left(L_{a c q} \mid 2\right) & \ldots & Q\left(L_{a c q} \mid L_{p}-1\right)
\end{array}\right]^{T},
$$

so that we have

$$
\vec{y}_{L_{a c q}}=A \vec{y}_{L_{a c q}-1}=A^{L_{a c q}} \vec{y}_{0}
$$


where $\vec{y}_{0}=\left[\begin{array}{lllll}1 & 0 & 1 & \ldots & 1\end{array}\right]^{T}$ and $A$ is equal to the transition matrix of the transmission Markov chain $Z_{n}$, except for the elements of the second row and second column, which are set to 0 , i.e.

$$
A(i, j)=\left\{\begin{array}{ll}
0 & \text { if } i=1 \text { or } j=1 \\
p_{Z}(i, j) & \text { otherwise }
\end{array} .\right.
$$

Thanks to the structure of $A$, it turns out that (31) becomes

$$
\vec{y}_{L_{a c q}}=\left[\begin{array}{c}
(1-q)^{L_{a c q}} \\
0 \\
(1-q)^{\max \left(0, L_{a c q}-\left(L_{p}-3\right)\right)} \\
\vdots \\
(1-q)^{\max \left(0, L_{a c q}-2\right)} \\
(1-q)^{\max \left(0, L_{a c q}-1\right)}
\end{array}\right] .
$$

Finally, putting (27), (30), and (32) together, $Q\left(L_{a c q}\right)$ can be calculated with

$$
Q\left(L_{a c q}\right)=\frac{(1-q)^{L_{a c q}}+q \sum_{i=1}^{L_{p}-3}(1-q)^{\max \left(0, L_{a c q}-i\right)}}{1+q\left(L_{p}-1\right)} .
$$

\section{Summary: how to use our method to compute the saturation throughput}

The saturation throughput $\lambda_{0}$ is obtained by solving Equation (1) numerically and Equation (1) is the combination of two subproblems. The first subproblem corresponds to modeling the MAC protocol: we have to compute $\left[\lambda_{0}, \lambda\right]=f\left(p_{\text {acq }}\right)$, by using Equations (15) and (16) in Section III-A. Essentially, for a given MAC protocol, we abstract the underlying retransmission Markov chain $X_{n}$ and compute its stationary distribution $\pi_{X}$. Then, we compute the durations of $t_{\mathrm{tx}}, t_{\mathrm{acq}}$, $t_{\text {fail }}(i), t_{\text {drop }}$ (defined in Section III-A4). Those values depend on the rate of the underlying physical layer, on the packet structure and working of the protocol.

For the second subproblem, specifically computing $p_{\text {acq }}=$ $g\left(\lambda_{0}, \lambda\right)$, it is solved by starting from (17) with (21), (22) and (18) with (33) in Section III-B. Because equations (17), (21), and (22) all depend on $p_{\text {acq }}$, we have to solve a quadratic system of equations in order to obtain $P_{\text {busy }}$ and $p_{\text {acq. }}$. This aforementioned set of equation models generically a physical layer with multi-channel capability. Equations (18) and (22) depend on the numerical values of $P_{\mathrm{MD}}^{(k, i)}$ and $\Theta$, respectively. Those values are obtained by numerical simulations of the packet detection and timing acquisition algorithm at the physical layer. Hence, to use our method with another packet detection and timing acquisition algorithm than [12] requires a physical layer implementation and extensive simulations to obtain the $P_{\mathrm{MD}}^{(k, i)}$ and $\Theta$ for this specific algorithm. Modeling multiple frequency bands should also be possible because transmitters on another frequency band can be modeled as in Equation (18) for the case of stations transmitting with another acquisition preamble than the destination.

\section{Performance evaluation}

In this section, we evaluate the performance of an IRUWB network. In particular, we compare the performance of a network that uses a common acquisition preamble with the performance of a network that uses private acquisition preambles. First, we use the analytical model derived previously to obtain performance results for a symmetric and homogeneous IR-UWB network. In this case, our performance metric is the saturation throughput $\lambda_{0}$. Second, we turn to ns-2 [11] simulations to address more general scenarios with single-hop and multi-hop topologies and UDP or TCP as the transport protocol.

\section{A. Scenarios and parameters of the performance evaluation}

The MAC protocol that is used in the modeling is the DCCMAC protocol. We already described it briefly in Section II-B. For more details on the protocol, the reader can refer to [6]. But, compared to the original specification in [6], the parameters of DCC-MAC have been adapted for an IEEE 802.15.4a type of network. In particular, the maximum physical layer bitrate is $1 \mathrm{Mbit} / \mathrm{s}$ and the maximum range is around 50 meters.

1) Parameters and scenarios for the analytical model: The fixed point equation (1) is solved numerically. The code for the fixed point problem is implemented with Matlab; the source code is available online [14]. For $t_{\mathrm{acq}}, t_{\mathrm{tx}}, t_{\text {fail }}(i), i=1, \ldots, R$ and $t_{\text {drop }}$ in the equations (8), we have the following values:

- The acquisition time $t_{\mathrm{acq}}$ corresponds to the length of the acquisition preamble. With the packet detection and timing acquisition algorithm in [12], the duration of the acquisition preamble is $64 \mu \mathrm{s}$.

- The transmission time $t_{\mathrm{tx}}$ is the sum of the following durations: the DATA packet duration (minus the length of the acquisition preamble), the round-trip time, the ACK packet duration, the SIGIDLE packet duration, and the maximum backoff time.

- The elapsed time in case of a failed packet transmission $t_{\text {fail }}(i)$ for $i=1, \ldots, R-1$ is the sum of the DATA packet duration, of the send timer, of the average duration of the idle timer (see [6]), and of the average backoff time in backoff stage $i$. For $i=R, t_{\text {fail }}(i)$ is only the sum of the DATA packet duration and of the send timer. For the average duration of the idle timer, it is obtained by extensive simulations of the DCC-MAC protocol. Indeed, the idle timer is stopped when a SIGIDLE packet is received from the destination of interest. Hence, its distribution and average value can only be obtained by simulation.

- In case of a packet drop, $t_{\mathrm{drop}}$ is the maximum backoff timer length.

For $t_{\mathrm{I}}$ and $t_{\mathrm{D}}$ in (21), we have the following values:

- The value of $t_{\mathrm{I}}$ depends on whether we do an early discard or not. For early discard, it is equal to the duration of an ACK packet transmission. For late discard, it is equal to the duration of a DATA packet.

- The value of $t_{\mathrm{D}}$ is equal to the duration of a DATA packet followed by the duration of an ACK packet.

- Moreover, in the case of common acquisition preambles, the duration of a SIGIDLE packet must also be added to $t_{\mathrm{I}}$ and $t_{\mathrm{D}}$. 


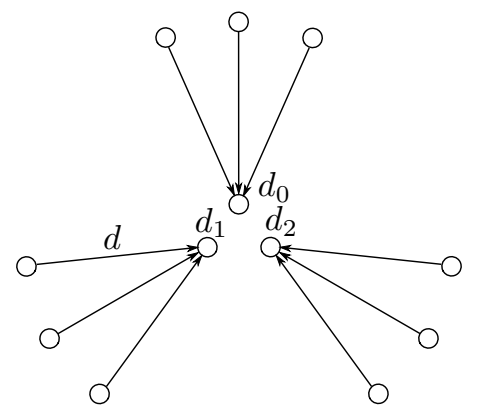

(a) Multiple piconets

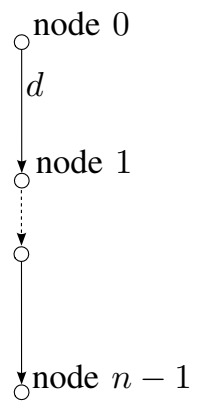

(b) Line topology

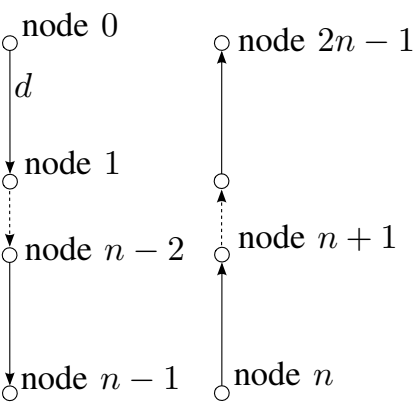

(c) Parallel lines topology

Fig. 3. Topologies used for the ns-2 simulations. The link distance is $d$.

The detailed numerical values can be found in the source code of the implementation of DCC-MAC or in the Matlab code solving the fixed point equation [14]. Values for $P_{\mathrm{MD}}^{(k, i)}$ in (18) are derived by extensive simulations from [12]. Values for $\Theta$ in (22) correspond to the probability of false alarm of the packet detection and timing acquisition scheme in [12].

To obtain the throughput from $\lambda_{0}$, we simply multiply $\lambda_{0}$ with the size of the payload of a packet; the throughput is

$$
\lambda_{0} P_{\text {packet }}
$$

where $P_{\text {packet }}$ is the payload of a packet in bits.

The scenario we analyze with our analytical model is a symmetric and homogeneous network. All destinations have the same number of identical sources, and the distance between the source and the destination is the same for all links.

2) Parameters and scenarios for the $n s-2$ simulations: The DCC-MAC protocol is implemented in ns-2 (release 2.29), along with an IR-UWB physical layer model. All details on the IR-UWB physical layer implementation can be found in [13]. In this paragraph, we give a short summary of how packet detection and timing acquisition is implemented in our ns-2 implementation. When a packet arrives at a destination, all further packets that arrive during the duration of the acquisition preamble are stored in a list. If a private acquisition preamble is used, we add to the list only the packets intended for the destination. In the case of a common acquisition preamble, we add all packets that arrive during the duration of the acquisition preamble to the list. At the end of the duration of the acquisition preamble, a packet in the list is chosen randomly (with a uniform distribution). This packet is further received by the physical layer with a probability $1-P_{\mathrm{MD}}^{(k, i)}$ where $k$ is the number of packets in the list (with the same acquisition preamble than the destination) and $i$ is the number of packets that have another acquisition preamble than the destination. Packets with a different acquisition preamble can create a false acquisition with a probability $\Theta$ (see (22)). For false acquisitions, we assume only late discard in the ns-2 simulations.

For our performance evaluation, we consider three different scenarios. Each scenario has a different topology and UDP or TCP as the transport protocol. The scenarios are the following:

- For the exposed piconets scenario, the topology consists of $n$ piconets with three sources and one destination per piconet (see Figure 3(a)). All nodes are in range of each other and all sources of a given piconet have the same destination inside the piconet. The distance between the sources and their destination is 10 meters. The distance between the respective destinations of the $n$ piconets is four meters. The transport protocol is UDP.

- For the TCP line scenario, the topology consists of a line of equidistant nodes (Figure 3(b)). The distance between neighboring nodes (or link distance) is 10 or 20 meters. The sender and the destination are placed at each extremity of the line. The transport protocol is TCP.

- For the parallel TCP lines scenario, the topology consists of two parallel lines of equidistant nodes (Figure 3(c)). The distance between neighboring nodes on a line is 10 or 20 meters and the distance between the two lines is always 20 meters. Each line has one source at one extremity and its associated destination at the other extremity. However, the two sources are not on the same extremity. The transport protocol is TCP.

Because the maximum range is around 50 meters, not all nodes are in range of each other in the TCP line and parallel TCP lines scenarios. For all scenarios with UDP, traffic is generated by a CBR source with a rate high enough to make sure that the lower layers are in saturated traffic conditions. For both UDP and TCP, the size of the payload $P_{\text {packet }}$ in (34) can be equal to $1000,500,250$, or 125 bytes. The topologies for the TCP line and the parallel TCP lines imply that multi-hop forwarding is used. Hence, for both scenarios, we configure static, multihop routes thanks to the NOAH routing agent in ns-2. Finally, for all results obtained with ns-2 simulations in Section IV-C, DCC-MAC runs with the rate adaptation algorithm enabled. For the validation of the analytical model in Section IV-B, a fixed rate is used. For every scenario, several simulation runs are performed. Each of them lasts 600 seconds and an initial transient duration of 100 seconds is ignored. We always show the $95 \%$ confidence interval for the median.

\section{B. Saturation throughput of a homogeneous IR-UWB network}

We first validate our analytical model. We compare the throughput obtained with our analytical model with ns-2 simulations with the exposed piconet scenario (but with one to 15 sources) and UDP traffic. In Figure 4, we plot the aggregate (sum of all sources) saturation throughput obtained with the analytical model and the ns- 2 simulations versus the 


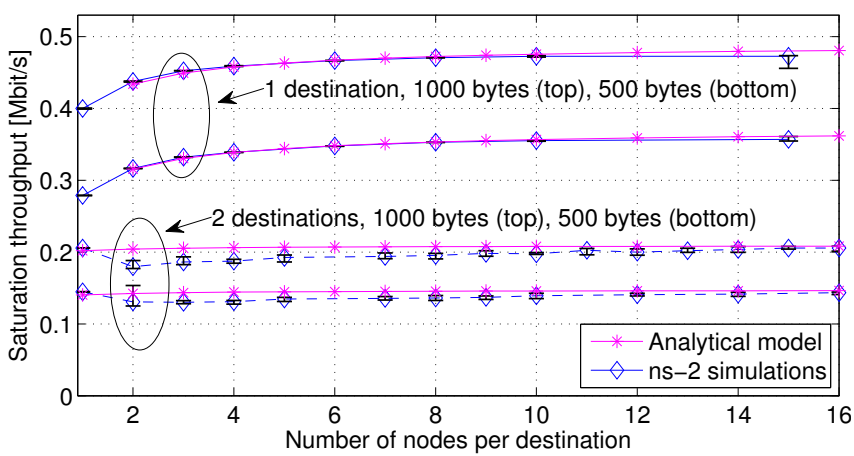

Fig. 4. Validation of the results obtained with the analytical model. The aggregate saturation throughput is plotted (sum of the throughput of all sources) versus the number of sources per destination $n$. The plain curve is the analytical saturation throughput, the dashed curve is the ns-2 simulations. We have results for a 1000 and 500 bytes payload. The upper pairs of curves are for one destination with $n$ transmitters and a private acquisition preamble. For the bottom pairs of curves, there is a second destination with $n$ concurrent transmitters and common acquisition preambles.

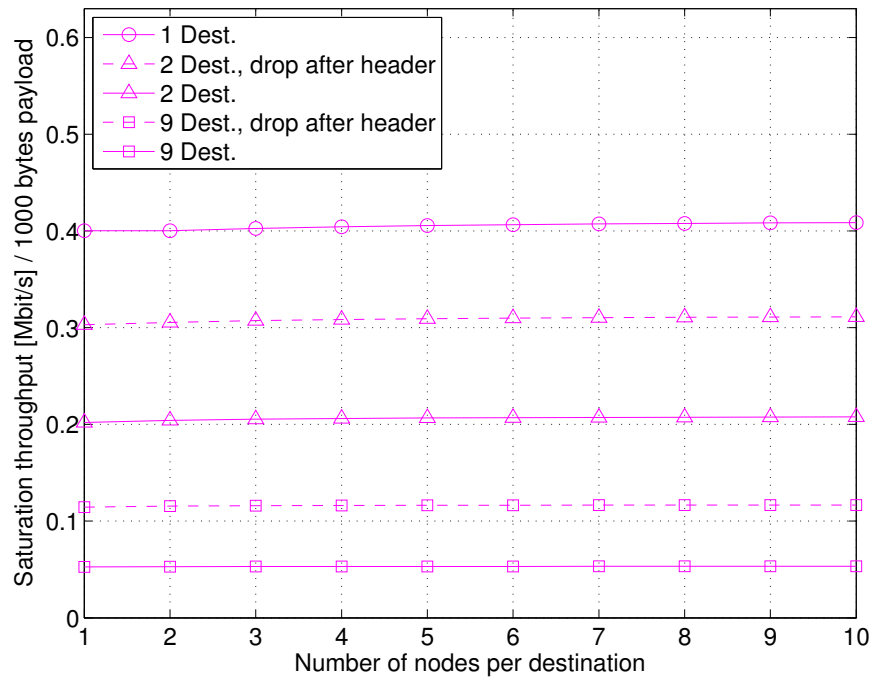

Fig. 5. The aggregate throughput (sum of the throughput of all sources) is plotted versus the number of nodes per destination $n$ for a 1000 bytes payload only. We have 1,2 and 9 destinations with a common acquisition preamble. There is a large drop in throughput when all sources use the same acquisition preamble. Early discard significantly increases the throughput. Results obtained with other (smaller) payload sizes are not shown because they lead to similar conclusions. The results with the private acquisition preamble are shown separately in Figure 6.

number of sources $n$ per destination. We consider one and two destinations and a payload size of 1000 or 500 bytes. Results with other payload sizes are not shown as they yield identical conclusions. As it can be observed, in both cases there is a slight discrepancy when the number of transmitters $n$ is small. This is expected as the mean-field assumption becomes valid for a large number of stations. The throughput decreases with a smaller payload size because the overhead per byte is larger.

For the the saturation throughput of a homogeneous IRUWB network, we look at three cases: one, two, and nine destinations and a payload of 1000 bytes. Results with other payload sizes are not shown as they yield identical conclusions. For each case, there are $n$ sources per destination. In

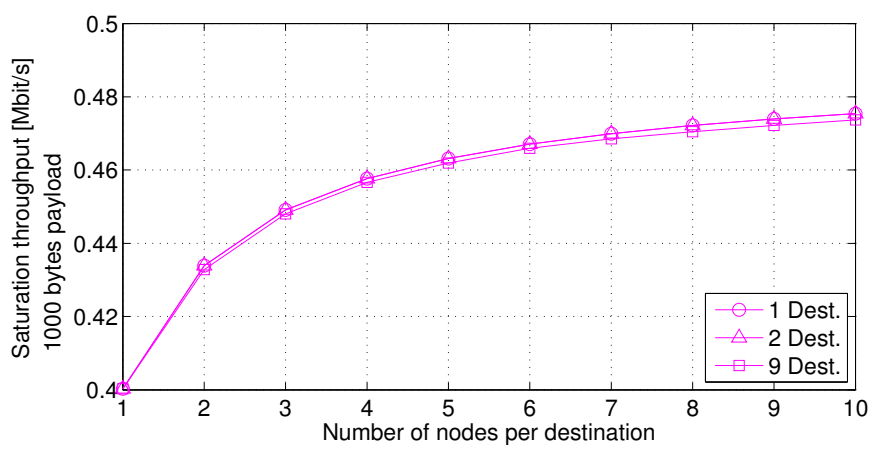

Fig. 6. The aggregate throughput (sum all throughputs) is plotted versus the number of nodes per destination $n$ for a 1000 bytes payload. We have 1,2 and 9 destinations with private acquisition preambles. There is a very small throughput degradation in the case of 9 destinations but no noticeable degradation in the case of 2 destinations.

Figure 5, we plot the aggregate saturation throughput versus $n$ for a network with a common acquisition preamble. The results with the private acquisition preamble are shown separately in Figure 6. The throughput is greatly reduced when all sources use the same acquisition preamble. Obviously, the throughput is notably increased when the packets that were unintentionally acquired are dropped as early as possible. In Figure 6, we also have one, two, and nine destinations but for a network with private acquisition preambles. There is a small reduction of the aggregate throughput with nine destinations compared to one destination. The results are all with late discard. With early discard, the results are not shown because they are not discernible from the aggregate throughput with one destination. The very small throughput difference comes from the robustness of the packet detection and timing acquisition algorithm in [12] and also, from the fact that we ignore interference on the payload part. All in all, these results clearly show the strong effect of packet detection and timing acquisition on the performance of an IR-UWB network if a common preamble is used.

\section{C. $n s-2$ simulations}

We now turn to ns-2 simulations for an evaluation with more realistic scenarios. We begin with the exposed piconets scenario (see Figure 3(a)).

1) Exposed piconets: For this scenario, remember that the number of sources per destination is fixed to three. In Figure 7 , we plot the aggregate saturation throughput per piconet versus the number of piconets (or equivalently the number of destinations) for payloads of 1000,500 and 250 bytes.

The throughput is strongly reduced when a common acquisition preamble is used. The difference between the throughput with a private acquisition preamble and a common acquisition preamble grows with the number of interferers. Furthermore, in the case of a common acquisition preamble, the confidence intervals have a larger width than in the case of private preambles. This indicates that, with a common preamble, there are throughput variations and instabilities between the different piconets. The throughput decreases with smaller payload sizes because the overhead per byte becomes larger. 


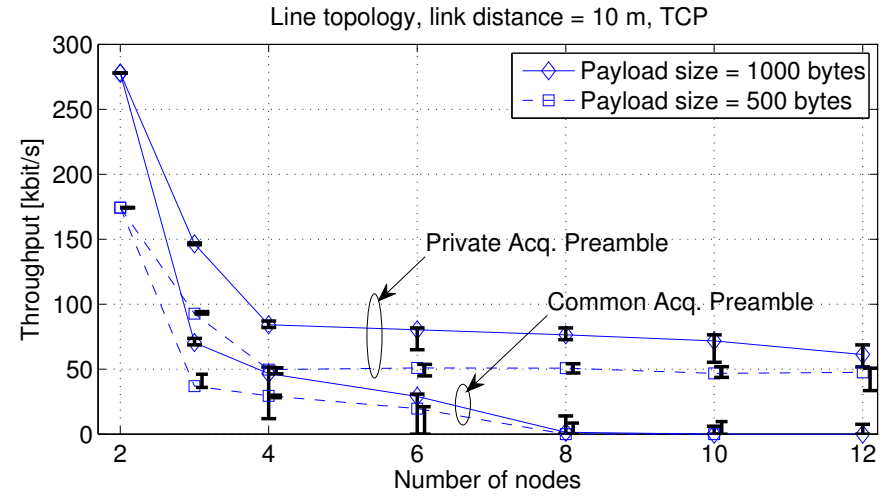

(a) 1000 and 500 bytes payload, 10 meters link distance

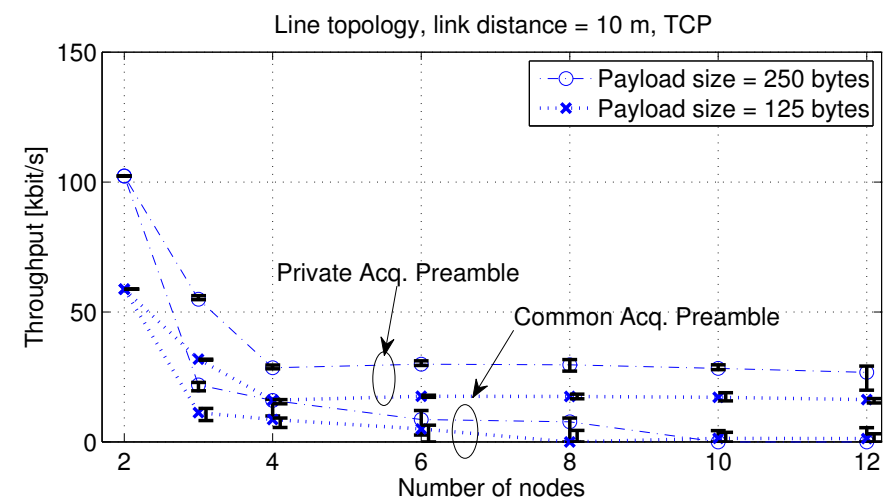

(c) 250 and 125 bytes payload, 10 meters link distance

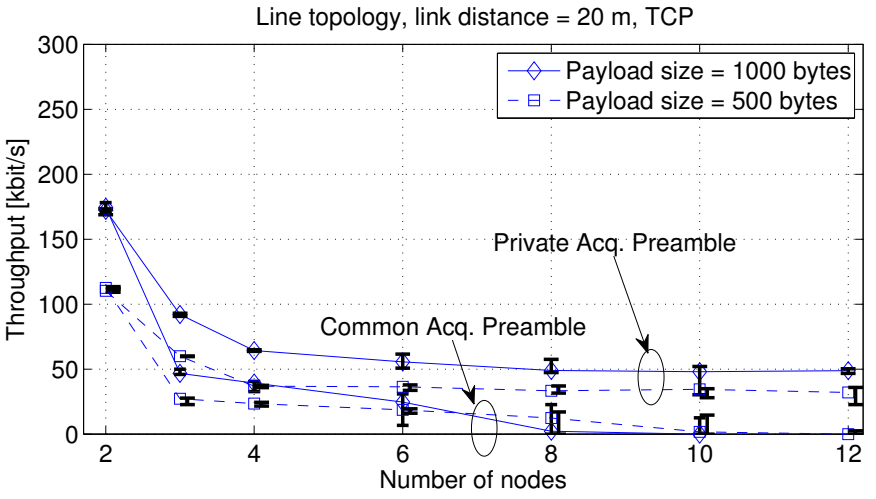

(b) 1000 and 500 bytes payload, 20 meters link distance

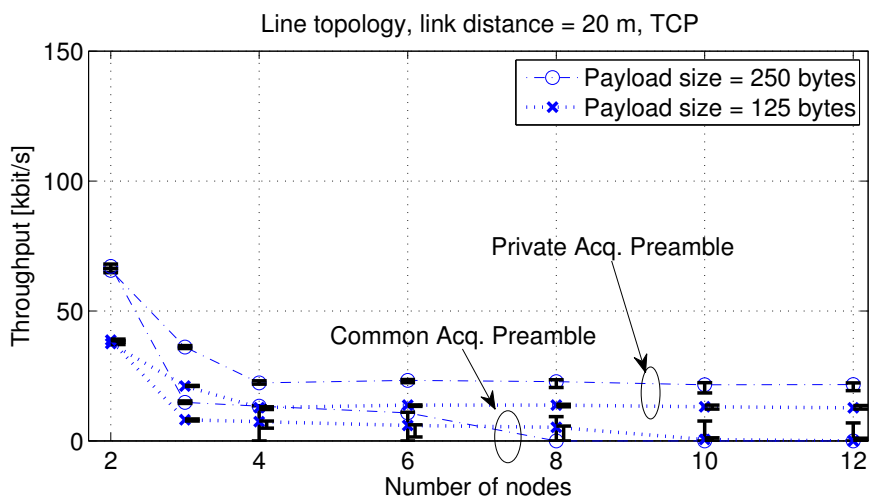

(d) 250 and 125 bytes payload, 20 meters link distance

Fig. 8. Line TCP scenario (see Figure 3(b)): throughput versus number of nodes for payloads of 1000,500 (top), 250 , and 125 (bottom) bytes. The link distance is 10 meters on the left column and 20 meters on the right column. The confidence intervals for the common acquisition preamble cases are slightly shifted to the right to discern them from the private acquisition preamble cases. This scenario shows a dramatic compounding effect where, in the case of common acquisition preambles, the throughput can drop to zero for more than six nodes. The network becomes also unstable when all sources use the same acquisition preamble. On the contrary, using a private acquisition preamble yields a stable and higher throughput.

2) TCP line and parallel TCP lines scenario: For the TCP line scenario, we plot the throughput of the source as a function of the number of nodes. For the parallel TCP lines, we show the results for the two sources (or flows) separately. For both topologies, there are two cases: a link distance of 10 or 20 meters. In addition, we have four different payload sizes of $1000,500,250$, and 125 bytes.

In the TCP line scenario (Figure 8), we can observe that a stable throughput is reached for more than four nodes with the use of private acquisition preambles. However, we observe a dramatic throughput reduction when all nodes use the same acquisition preamble. Indeed, for more than six nodes, the throughput reaches zero for some simulation run. And in the case of ten nodes or more, the network does not function at all. In addition, as indicated by the width of the confidence intervals, there is much more variability in the network behavior with a common preamble than with private acquisition preambles.

In Figure 9, even more severe effects are observed when using a common acquisition preamble in the case of the parallel TCP lines scenario. There are several plots for various payload sizes and for both link distances of 10 and 20 meters.
We always plot the throughput of flow 1 slightly shifted to the left and that of flow 2 shifted to the the right. For the common acquisition preamble case, we observe an almost complete collapse of the network when the number of nodes is larger than six (i.e. more than two hops), especially for a 1000 bytes payload. In addition, there is a notable unfairness between the two TCP flows. Furthermore, the throughput variations are much larger with the smaller link distance of 10 meters. On the contrary, the use of private acquisition preambles allow for a much higher and more stable throughput. With smaller payload sizes of 500 and 250 bytes, the network tends to be a bit more stable but the throughput also decreases. The results for a 125 bytes payload are not shown but lead to identical conclusions. The unfairness that we observe is very similar to what happens in IEEE 802.11 networks in exposed node cases [24].

\section{Validity and applicability of our results}

The interference model for the payload that we currently use in the ns-2 implementation of the IR-UWB physical layer is rather optimistic [13]. It corresponds to the use of an optimal Rake receiver at the physical layer. Hence, the collapse 


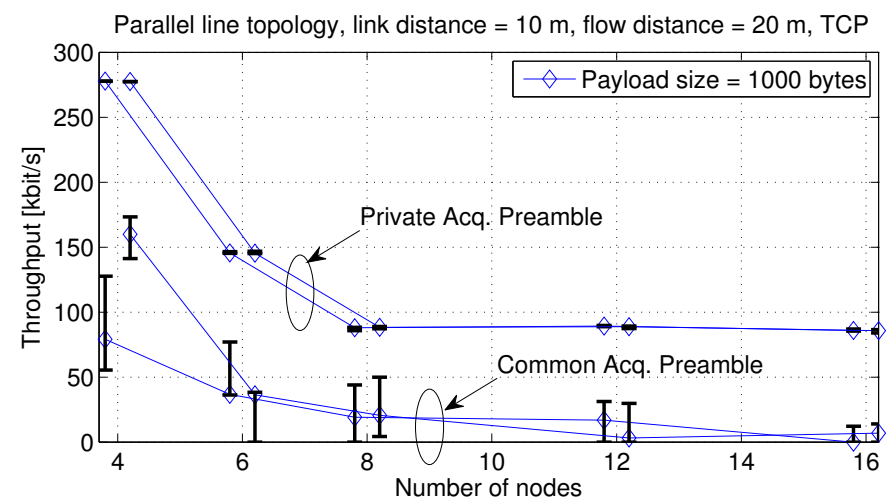

(a) 1000 bytes payload, 10 meters link distance

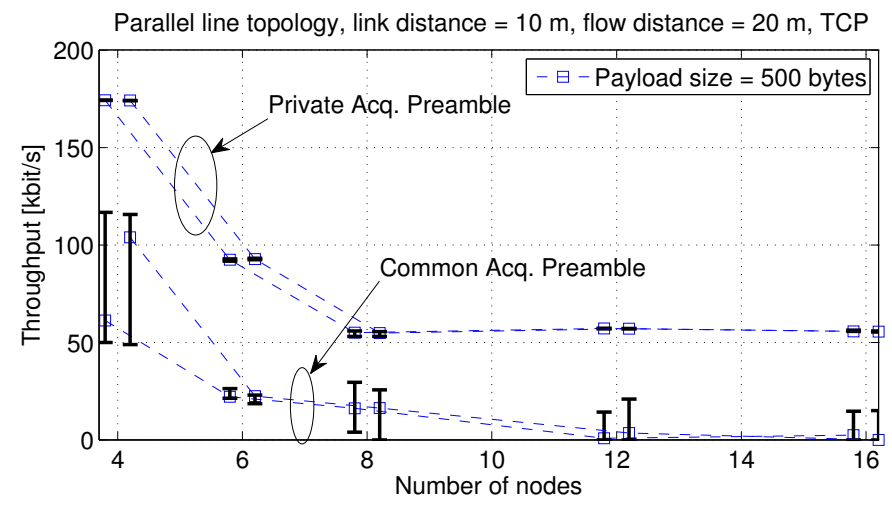

(c) 500 bytes payload, 10 meters link distance

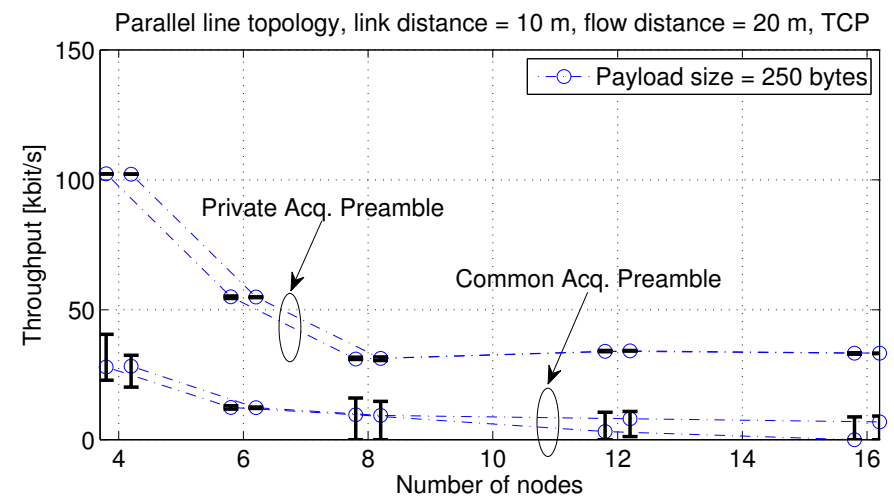

(e) 250 bytes payload, 10 meters link distance

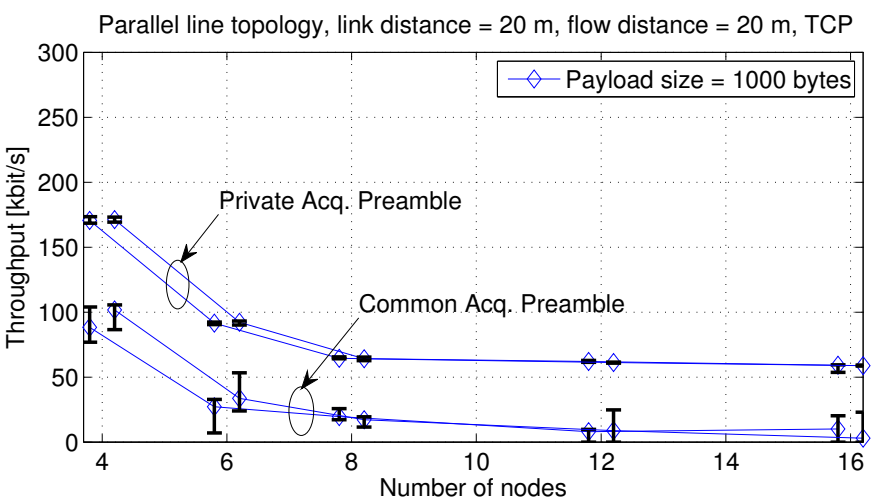

(b) 1000 bytes payload, 20 meters link distance

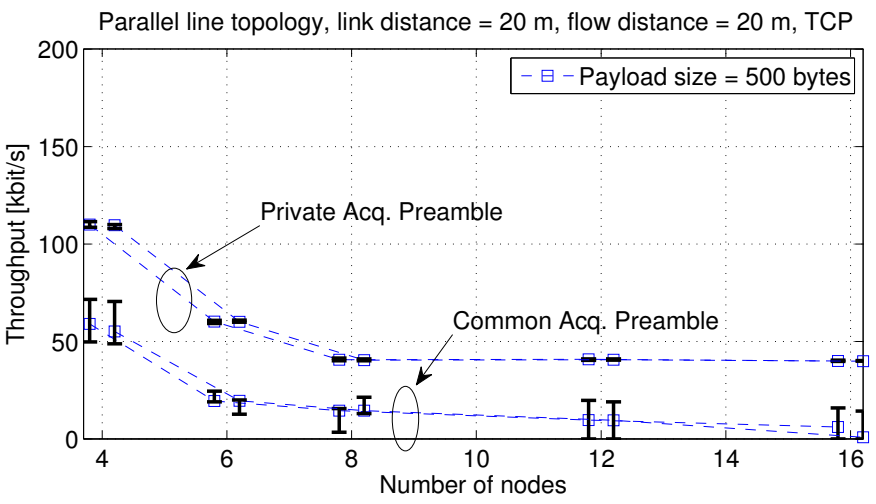

(d) 500 bytes payload, 20 meters link distance

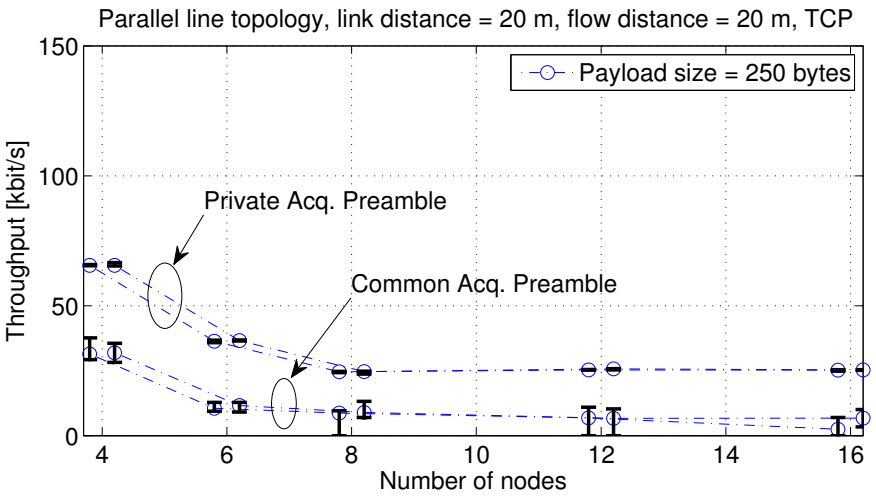

(f) 250 bytes payload, 20 meters link distance

Fig. 9. Parallel TCP lines scenario (see Figure 3(c)): throughput per flow versus number of nodes for 1000 (top), 500 (middle) and 250 (bottom) bytes. The link distance is 10 meters on the left column and 20 meters on the right column. For each figure, the results for flow 1 are slightly shifted to the left, and those for flow 2 are slightly shifted to the right. This scenario shows a dramatic compounding effect where, in the case of a common acquisition preamble, the network can completely collapse for more than two hops. A high unfairness between the two flows can also be observed with a common acquisition preamble. With smaller payload sizes, the network tends to be a bit more stable but the throughput decreases. On the contrary, using a private acquisition preamble yields a stable and higher throughput. 


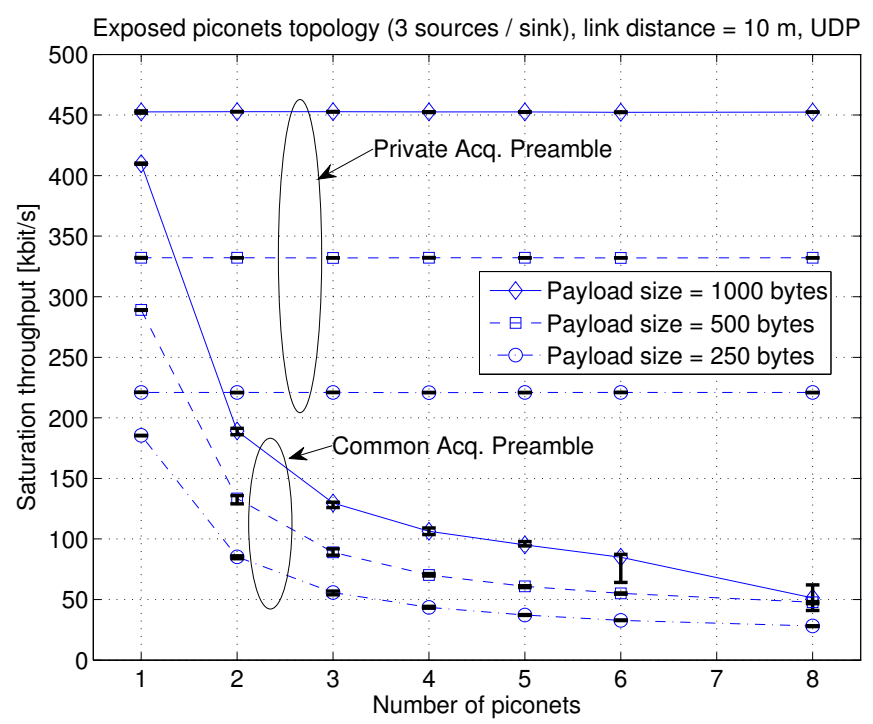

Fig. 7. Exposed piconets scenario (see Figure 3(a)): aggregate saturation throughput per piconet versus the number of piconets for payloads of 1000 , 500 and 250 bytes. The difference between the throughput with a private acquisition preamble and a common acquisition preamble grows with the number of interferers. In addition, the network can be less stable when all sources use the same acquisition preamble as indicated by the larger confidence interval values. The throughput decreases with smaller payload sizes because the overhead per byte becomes larger.

of the network observed in the line TCP and parallel lines TCP scenarios results strictly from the fact that a common acquisition preamble is used for packet detection and timing acquisition. For packet detection and timing acquisition, MUI is taken into account indirectly through the numerical values of $P_{\mathrm{MD}}^{(k, i)}$ and $\Theta$ : The physical layer simulations performed to obtain those numerical values take into account signal collisions because of multipath propagation and asynchronous transmissions.

In the case another MAC protocol than DCC-MAC would be used for the performance evaluation, we believe our results would still hold. For instance, the MAC protocols for the IEEE 802.15.4a standard are Aloha or CSMA/CA with an optional RTS/CTS exchange. DCC-MAC has no RTS/CTS control packet but uses a SIGIDLE control packet instead (Section II-B). In any case, for every transmitted packet, packet detection and timing acquisition is currently necessary for the aforementioned protocols, so the throughput definitely depends on how packet detection and timing acquisition is performed. Hence, for Aloha or CSMA/CA, a private acquisition preamble will still offer a large throughput gain compared to a common acquisition preamble.

\section{RELATED WORK}

The IR-UWB physical layer with time-hopping is described in [2] and with greater details in [25]. For more information on the IR-UWB physical layer the reader can consult [26], [27], [28] and the references therein.

The work in [18] and later in [19] addresses the optimal design of IR-UWB networks. Some of the main findings in [18] are that a MAC protocol for IR-UWB networks should not use power control and should not use mutual exclusion, but rather should allow sources to transmit whenever they want, as long as they are outside an exclusion region around destinations. The interference created by the concurrent transmissions should be managed with rate adaptation. It was established later in [6] that the size of the exclusion region for low datarate IR-UWB networks is negligible.

There is a large body of work on practical MAC protocols for IR-UWB networks [6], [7], [29], [30], [31], [32], [33], [34]. We have already discussed DCC-MAC [6] in Section II-B. Still, it is interesting to observe that DCCMAC is actually quite close to the mandatory MAC protocol in the IEEE 802.15.4a amendment [3] with the following notable exceptions: in IEEE 802.15.4a a common acquisition preamble is used, there is no rate adaptation and no SIGIDLE packet. $(\mathrm{UWB})^{2}$ is the MAC protocol described in [7]. As for DCC-MAC, private acquisition preambles are used. But, the private acquisition preambles are chosen dynamically for each packet with a prior exchange of control packets on a control channel. The control channel is implemented thanks to a common acquisition preamble. The MAC protocol presented in [30] is called U-MAC. U-MAC also uses private acquisition preambles and tries to set optimally both the rate and transmit power for each packet transmission. The work in [31] assumes that a specific hardware provides a way to detect activity on the medium for an IR-UWB physical layer. Then it proposes a CSMA based MAC protocol with a busy tone approach [35] and common acquisition preambles. In [32], a MAC protocol based on mutual exclusion [18] is proposed for a single-hop network.

The MAC protocols in [33] and [34] explore the additional use of multiple UWB frequency bands. For instance, instead of transmitting an IR-UWB signal over a frequency band of $5 \mathrm{GHz}, 10$ bands of $500 \mathrm{MHz}$ could be used. And it appears in [34] that a multiple band approach may offer a better throughput than a single band approach.

There is a survey of MAC protocols for IR-UWB networks in [29] with additional references therein. All the MAC protocols presented in the previous paragraph could replace DCC-MAC in our analytical model. In particular, modeling multiple frequency bands, in addition to the effect of the acquisition preamble, appears interesting. In [36], models of the interference created by interference on different cochannels are presented, although for narrow-band physical layers. Also, the IR-UWB physical layer is actually a multichannel physical layer. There are several papers that discuss MAC protocols for multi-channel narrow-band physical layers. The reader can start with [37] and the references therein.

The approach we use for our analytical model with a fixedpoint equation is similar to previous work in [9], [21], [10]. Indeed, a mean-field assumption (or decoupling assumption) is done in [21], [10]. And [9] relies on a similar independence hypothesis. However, our work is different in that it takes into account an IR-UWB physical layer that has different properties than the narrow-band physical layer assumed in the previous work. Moreover, we explicitly address packet detection and timing acquisition and study their effect on the network. To the best of our knowledge, packet detection and timing acquisition 
are ignored in the previous work on networking.

\section{CONCLuSion}

We have analyzed how using a private or common acquisition preamble affects the performance of IR-UWB networks. We have developed an analytical model to compute the saturation throughput of an IR-UWB network. One novelty of our model compared to previous work is that it explicitly takes into account packet detection and timing acquisition. Our model can be used with different MAC layers or different acquisition algorithms. Using the model, we show that a private acquisition preamble offers a large throughput gain (larger than $100 \%$ ) compared to a common acquisition preamble. Moreover, the throughput difference grows with the number of concurrent transmitters and interferers. Finally, with ns2 simulations on multi-hop topologies with TCP flows, we demonstrate that a network using private acquisition preambles exhibits a stable throughput. On the contrary, using a common acquisition preamble exhibits the presence of a compounding effect similar to the exposed terminal issue in IEEE 802.11 networks: the throughput is severely degraded and complete flow starvation might occur. Further, the use of a common acquisition preamble results in very large performance fluctuations in some scenarios. Future work should explicitly take into account the cost of learning the acquisition preamble of a destination. Also, a proper modeling of the interference on the payload is necessary.

\section{ACKNOWLEDGEMENTS}

The authors would like to thank Saravanan Vijayakumaran for helpful discussions and his contribution early in the work of this article.

\section{REFERENCES}

[1] R. Merz, J.-Y. Le Boudec, and S. Vijayakumaran, "Effect on network performance of common versus private acquisition sequences for impulse radio uwb networks," in ICUWB 06, September 2006.

[2] M. Z. Win and R. A. Scholtz, "Impulse radio: how it works," IEEE Commun. Lett., vol. 2, no. 2, pp. 36-38, 1998.

[3] IEEE Computer Society, LAN/MAC Standard Committee, "IEEE P802.15.4a/D7 (amendment of IEEE std 802.15.4), part 15.4: Wireless medium access control (MAC) and physical layer (PHY) specifications for low-rate wireless personal area networks," Jan. 2007.

[4] — , "IEEE standard for information technology- telecommunications and information exchange between systems- local and metropolitan area networks- specific requirements part 15.4: Wireless medium access control (MAC) and physical layer (PHY) specifications for low-rate wireless personal area networks (WPANs)," IEEE Std 802.15.4-2006 (Revision of IEEE Std 802.15.4-2003), 2006.

[5] E. Callaway, P. Gorday, L. Hester, J. Gutierrez, M. Naeve, B. Heile, and V. Bahl, "Home networking with IEEE 802.15.4: a developing standard for low-rate wireless personal area networks," IEEE Commun. Mag., vol. 40, no. 8, pp. 70-77, August 2002.

[6] R. Merz, J. Widmer, J.-Y. Le Boudec, and B. Radunovic, "A joint PHY/MAC architecture for low-radiated power TH-UWB wireless adhoc networks," Wireless Commun. and Mobile Comput. J., vol. 5, no. 5, pp. 567-580, August 2005.

[7] M.-G. Di Benedetto, L. Nardis, M. Junk, and G. Giancola, "(UWB $)^{2}$ : Uncoordinated, wireless, baseborn, medium access control for UWB communication networks," Mobile Networks and Applications, vol. 10, no. 5, October 2005.

[8] L. Chalard, D. Helal, L. Verbaere, A. Wellig, and J. Zory, "Wireless sensor networks devices: Overview, issues, state-of the art and promising technologies," ST Journal of Research, Wireless Sensor Networks, vol. 4, no. 1, pp. 4-18, April 2007.
[9] G. Bianchi, "Performance analysis of the IEEE 802.11 distributed coordination function," IEEE J. Sel. Areas Commun., vol. 18, no. 3 , pp. 535-547, March 2000.

[10] A. Kumar, E. Altman, D. Miorandi, and M. Goyal, "New insights from a fixed-point analysis of single cell ieee 802.11 wlans," IEEE/ACM Trans. Netw., vol. 15, no. 3, pp. 588-601, 2007.

[11] “The network simulator ns-2, http://nsnam.isi.edu/nsnam," January 2008.

[12] A. El Fawal and J.-Y. Le Boudec, "A robust signal detection method for ultra wide band (UWB) networks with uncontrolled interference," IEEE Trans. Microw. Theory Tech., vol. 54, no. 4, pp. 1769-1781, June 2006.

[13] R. Merz, J.-Y. Le Boudec, and J. Widmer, "An architecture for wireless simulation in NS-2 applied to impulse-radio ultra-wide band networks," in 10th Communications and Networking Simulation Symposium, 2007.

[14] "UWB research at EPFL-IC," http://icawww1.epfl.ch/uwb/, 2008.

[15] J. G. Proakis, Digital Communications, 4th ed., 2001.

[16] H. Hashemi, "The indoor radio propagation channel," Proc. IEEE, vol. 81, no. 7, pp. 943-968, 1993.

[17] M. Z. Win and R. A. Scholtz, "On the robustness of ultra-wide bandwidth signals in dense multipath environments," IEEE Commun. Lett., vol. 2, no. 2, pp. 51-53, 1998.

[18] B. Radunovic and J. Y. Le Boudec, "Optimal power control, scheduling and routing in UWB networks," IEEE J. Sel. Areas Commun., vol. 22, no. 7, pp. 1252-1270, September 2004.

[19] A. El Fawal, J.-Y. Le Boudec, R. Merz, B. Radunovic, J. Widmer, and G. M. Maggio, "Tradeoff analysis of PHY-aware MAC in low-rate, lowpower UWB networks," IEEE Commun. Mag., vol. 43, no. 12, 2005.

[20] B. Crow, I. Widjaja, L. Kim, and P. Sakai, "IEEE 802.11 wireless local area networks," IEEE Commun. Mag., vol. 35, no. 9, September 1997.

[21] C. Bordenave, D. Mc Donald, and A. Proutière, "Random multiaccess algorithms, a mean field analysis," in Forty-third annual Allerton conference on Communication, Control, and Computing, 2005.

[22] F. Baccelli and P. Brémaud, Palm Probabilities and Stationary Queues. Springer Verlag Lecture Notes in Statistics, March 1987.

[23] J.-Y. Le Boudec, "Performance evaluation lecture notes," available at http://icawww1.epfl.ch/perfeval, 2007.

[24] K. Xu, M. Gerla, L. Qi, and Y. Shu, "Enhancing TCP fairness in ad hoc wireless networks using neighborhood RED," in MobiCom 03, 2003.

[25] M. Z. Win and R. A. Scholtz, "Ultra-wide bandwidth time-hopping spread-spectrum impulse radio for wireless multiple-access communications," IEEE Trans. Commun., vol. 48, no. 4, pp. 679-691, April 2000.

[26] L. Yang and G. B. Giannakis, "Ultra-wideband communications: an idea whose time has come," IEEE Signal Process. Mag., vol. 21, no. 6, 2004.

[27] R. C. Qiu, H. Liu, and X. Shen, "Ultra-wideband for multiple access communications," IEEE Commun. Mag., vol. 43, no. 2, pp. 80-87, 2005.

[28] D. D. Wentzloff, R. Blazquez, F. S. Lee, B. P. Ginsburg, J. Powell, and A. P. Chandrakasan, "System design considerations for ultra-wideband communication," IEEE Commun. Mag., vol. 43, no. 8, 2005.

[29] X. Shen, W. Zhuang, H. Jiang, and J. Cai, "Medium access control in ultra-wideband wireless networks," IEEE Trans. Veh. Technol., vol. 54, no. 5, pp. 1663-1677, 2005.

[30] R. Jurdak, P. Baldi, and C. V. Lopes, "U-mac: a proactive and adaptive uwb medium access control protocol," Wireless Commun. and Mobile Comput. J., Special Issue on Ultrawideband (UWB) Communications, vol. 5, no. 5, pp. 551-566, August 2005.

[31] N. J. August, H. J. Lee, and D. S. Ha, "Enabling distributed medium access control for impulse-based ultrawideband radios," IEEE Trans. Veh. Technol., vol. 56, no. 3, pp. 1064-1075, 2007.

[32] N. Shi, L. Xia, and I. Niemegeers, "A novel approach for the link layer in impulse-based uwb ad hoc networks," Wireless Personal Communications, vol. 42, no. 2, pp. 143-159, July 2007.

[33] I. Broustis, M. Molle, S. Krishnamurthy, M. Faloutsos, and J. Foerster, "A new binary conflict resolution-based mac protocol for impulse-based uwb ad hoc networks," Wireless Commun. and Mobile Comput. J., vol. 6, no. 7, pp. 933-949, 2006.

[34] I. Broustis, S. V. Krishnamurthy, M. Faloutsos, M. Molle, and J. R. Foerster, "Multiband media access control in impulse-based uwb ad hoc networks," IEEE Trans. Mobile Comput., vol. 6, no. 4, 2007.

[35] F. Tobagi and L. Kleinrock, "Packet switching in radio channels: Part ii-the hidden terminal problem in carrier sense multiple-access and the busy-tone solution," IEEE Trans. Commun., vol. 23, no. 12, 1975.

[36] A. Mishra, V. Shrivastava, S. Banerjee, and W. Arbaugh, "Partially overlapped channels not considered harmful," in SIGMETRICS 06.

[37] J. Mo, S. Wilson, and J. Walrand, "Comparison of multichannel mac protocols," Transactions on Mobile Computing, vol. 7, no. 1, 2008. 